\title{
Multi-loop Integrand Reduction via Multivariate Polynomial Division
}

\author{
Hans van Deurzen, Gionata Luisoni, Edoardo Mirabella, Tiziano Peraro*, \\ Ulrich Schubert \\ Max-Planck Insitut für Physik, München, Germany \\ E-mail: \{hdeurzen, luisonig, mirabell, peraro, schubert\} @mpp.mpg.de
}

\section{Pierpaolo Mastrolia}

Max-Planck Insitut für Physik, München, Germany

Dipartimento di Fisica e Astronomia, Università di Padova, and INFN Sezione di Padova, Italy

E-mail: ppaolo@mpp.mpg • de

\section{Giovanni Ossola}

Physics Department, New York City College of Technology, The City University of New York, USA

The Graduate School and University Center, The City University of New York, USA

E-mail: gossolaecitytech. cuny .edu

\begin{abstract}
We present recent developments on the topic of the integrand reduction of scattering amplitudes. Integrand-level methods allow to express an amplitude as a linear combination of Master Integrals, by performing operations on the corresponding integrands. This approach has already been successfully applied and automated at one loop, and recently extended to higher loops. We describe a coherent framework based on simple concepts of algebraic geometry, such as multivariate polynomial division, which can be used in order to obtain the integrand decomposition of any amplitude at any loop order. In the one-loop case, we discuss an improved reduction algorithm, based on the application of the Laurent series expansion to the integrands, which has been implemented in the semi-numerical library NINJA. At two loops, we present the reduction of five-point amplitudes in $\mathscr{N}=4 \mathrm{SYM}$, with a unitarity-based construction of the integrand. We also describe the multi-loop divide-and-conquer approach, which can always be used to find the integrand decomposition of any Feynman graph, regardless of the form and the complexity of the integrand, with purely algebraic operations.
\end{abstract}

11th International Symposium on Radiative Corrections (Applications of Quantum Field Theory to Phenomenology)

22-27 September 2013

Lumley Castle Hotel, Durham, UK

${ }^{*}$ Speaker. 


\section{Introduction}

Scattering amplitudes are analytic functions of the kinematic variables. The investigation of the multi-particle factorization properties fulfilled at their singular points [1-5], lead to important achievements in the development of methods for their computation. Integrand reduction methods, developed for one-loop diagrams [6,7] and recently extended to higher loops [8-12], allow to express scattering amplitudes as linear combinations of Master Integrals (MI's), by exploiting the knowledge of the analytic and algebraic structure of their integrands.

The integrand of a Feynman diagram is a rational function of the components of the loop momenta, namely a polynomial numerator sitting over a set of quadratic loop denominators corresponding to internal propagators. Any integrand can be written as a combination of fundamental, irreducible contributions. These contributions are integrands characterized by a subset of the original denominators and an irreducible polynomial residue for numerator. The multiple-cut conditions, which put these loop-momenta simultaneously on-shell, can be viewed as projectors isolating the corresponding residue. The residues have a universal process-independent parametric form. Some of the unknown process-dependent coefficients appearing in this parametrization can be identified with the ones which multiply the Master Integrals.

In Refs. $[10,11]$ the determination of the residues at the multiple cuts has been formulated as a problem of multivariate polynomial division, and solved at any loop order using algebraicgeometry techniques. The most general parametric form of a residue is indeed the most general remainder of a polynomial division modulo the ideal generated by the corresponding subset of denominators. The unknown coefficients of this parametrization can then be computed, within the fit-on-the-cut approach, by evaluating the integrand on values of the loop momenta such that the loop denominators which identify the residue are on-shell, as traditionally done in the one-loop case. In this paper, we also present a two-loop example in $\mathscr{N}=4 \mathrm{SYM}$.

The evaluation of the coefficients of the MI's of a one-loop amplitudes can be improved by performing a Laurent expansion with respect to the variables which are not fixed by the cut conditions, as we showed in Ref. [13]. The algorithm has been implemented in the semi-numerical C++ library NINJA, which proved to be faster and numerically more stable than the original integrandreduction approach. The library has been used for the computation of NLO QCD corrections to Higgs boson production in association with a top quark pair and a jet [14].

One can also perform the full integrand decomposition of any multi-loop amplitude by means of purely algebraic operations, within what we call the divide-and-conquer approach [12]. In this case, the residues are generated by performing successive polynomial divisions between the numerator and the denominators of a diagram. Because of its wider range of applicability we may consider the latter a more general method for the integrand decomposition of loop integrals.

\section{Integrand reduction formula}

An arbitrary $\ell$-loop graph represents a $d$-dimensional integral of the form

$$
\int d^{d} q_{1} \cdots d^{d} q_{\ell} \mathscr{I}_{i_{1} \cdots i_{n}}, \quad \quad \mathscr{I}_{i_{1} \cdots i_{n}} \equiv \frac{\mathscr{N}_{i_{1} \cdots i_{n}}}{D_{i_{1}} \cdots D_{i_{n}}}
$$


where $i_{1}, \ldots, i_{n}$ are (not necessarily distinct) indices labeling loop propagators. The numerator $\mathscr{N}$ and the denominators $D_{i}$ are polynomials in a set of coordinates $\mathbf{z}$, which correspond to the components of the loop momenta with respect to some basis. Let $P[\mathbf{z}]$ be the ring of all polynomials in such coordinates. Every set of indices $\left\{i_{1}, \ldots, i_{n}\right\}$ defines the ideal

$$
\mathscr{J}_{i_{1} i_{2} \cdots i_{n}} \equiv\left\langle D_{i_{1}}, \ldots, D_{i_{n}}\right\rangle=\left\{\sum_{k=1}^{n} h_{k}(\mathbf{z}) D_{i_{k}}(\mathbf{z}): h_{k}(\mathbf{z}) \in P[\mathbf{z}]\right\} .
$$

The goal of the integrand reduction is finding a decomposition of the form

$$
\mathscr{I}_{i_{1} \cdots i_{n}} \equiv \frac{\mathscr{N}_{i_{1} \cdots i_{n}}}{D_{i_{1}} \cdots D_{i_{n}}}=\sum_{k=0}^{n} \sum_{\left\{j_{1} \cdots j_{k}\right\}} \frac{\Delta_{j_{1} \cdots j_{k}}}{D_{j_{1}} \cdots D_{j_{k}}}
$$

where the residues $\Delta_{j_{1} \cdots j_{k}}$ are irreducible polynomials, i.e. polynomials which contain no contribution belonging in the corresponding ideal $\mathscr{J}_{i_{1} \cdots i_{n}}$.

The numerator $\mathscr{N}$ of the integrand can be decomposed by performing the multivariate polynomial division modulo a Gröbner basis $\mathscr{G}_{i_{1} \cdots i_{n}}$ of $\mathscr{J}_{i_{1} \cdots i_{n}}$ as

$$
\mathscr{N}_{i_{1} \cdots i_{n}}=\Gamma_{i_{1} \cdots i_{n}}+\Delta_{i_{1} \cdots i_{n}}=\sum_{k=1}^{n} \mathscr{N}_{i_{1} \cdots i_{k-1} i_{k+1} \cdots i_{n}} D_{i_{k}}+\Delta_{i_{1} \cdots i_{n}}
$$

in terms of a quotient $\Gamma_{i_{1} \cdots i_{n}}$ and the remainder $\Delta_{i_{1} \cdots i_{n}}$. The properties of Gröbner bases ensure that the remainder is irreducible, therefore it is identified with the residue of the multiple cut $D_{i_{1}}=\cdots=$ $D_{i_{n}}=0$, as suggested by the notation. The quotient $\Gamma$ belongs instead to the ideal $\mathscr{J}$, hence in the last equality of Eq. (2.3) it has been written as combination of denominators. Substituting Eq. (2.3) in Eq. (2.1), we obtain the recursive formula $[11,12]$

$$
\mathscr{I}_{i_{1} \cdots i_{n}}=\sum_{k=1}^{n} \mathscr{I}_{i_{1} \cdots i_{k-1} i_{k+1} \cdots i_{n}}+\frac{\Delta_{i_{1} \cdots i_{n}}}{D_{i_{1}} \cdots D_{i_{n}}}
$$

Eq. (2.4) expresses a given integrand in terms of an irreducible residue sitting over its denominators and a sum of integrands corresponding to sub-diagrams with fewer loop propagators. Hence, the recursive application of this formula ultimately yields the full decomposition of any integrand in terms of irreducible residues and denominators, as in Eq. (2).

The existence of such a recursive formula proves that the integrand decomposition can be extended at any number of loops and the most general parametrization of a residue $\Delta_{i_{1} \cdots i_{n}}$ can be identified with the most general remainder of a polynomial division modulo the Gröbner basis $\mathscr{G}_{i_{1} \cdots i_{n}}$. Within the fit-on-the-cut approach the unknown coefficients of this parametrization can be found by evaluating the numerator on the solutions of the multiple cut $D_{i_{1}}=\cdots=D_{i_{n}}=0$. This method has been traditionally used at one loop, and recently applied to higher-loop amplitudes as well $[8,9,15-17]$.

In Ref. [11], we applied the recursive formula in Eq. (2.4) to the most general one-loop integrand. This allowed to easily derive the well know OPP decomposition for dimensionallyregulated one-loop amplitudes [6,7], as well as its higher-rank generalization for effective and non-renormalizable theories [13] implemented in XSAMURAI (which extends the SAMURAI library [18]) and recently used in the computation of NLO QCD corrections to Higgs boson production plus two [19] and three jets [20] in gluon fusion, in the infinite top-mass approximation. 


\section{Integrand-Reduction via Laurent Expansion with NINJA}

An improved integrand-reduction method for one-loop amplitudes was presented in [13], elaborating on the the techniques proposed in $[21,22]$. This method allows to compute the coefficients of the Master Integrals, through the systematic application of the Laurent series expansion on the integrand, with respect to one of the free components of the loop momenta which are not fixed by the on-shell conditions.

Within the original integrand reduction algorithm [18,23, 24], the determination of the unknown coefficients requires to sample the numerator on a finite subset of the on-shell solutions, subtract from the integrand all the non-vanishing contributions coming from higher-point residues, and solve the resulting linear system of equations. Since in the asymptotic limit both the integrand and the higher-point subtractions exhibit the same polynomial behavior as the residue, with the Laurent-expansion method one can instead identify the unknown coefficients with the ones of the expansion of the integrand, corrected by the contributions coming from higher-point residues. In other words, with this approach the system of equations for the coefficients becomes diagonal and the subtractions of higher-point contributions can be implemented as corrections at the coefficient level which replace the subtractions at the integrand level of the original algorithm. The parametric form of this corrections can be computed once and for all, in terms of a subset of the higher-point coefficients.

This reduction algorithm has been implemented in the semi-numerical C++ library NINJA, which has been interfaced with the package GoSAM [25-29] for automated one-loop computations. Since the integrand of a loop amplitude is a rational function, its semi-numerical Laurent expansion has been implemented as a simplified polynomial division between the numerator and the denominators.

The input of the algorithm implemented in NINJA is the numerator cast in four different forms, which can be easily and very quickly generated from the knowledge of the analytic dependence of the integrand on the loop momentum (e.g. from the analytic expression of the numerator generated by GoSAM). The first form corresponds to a simple evaluation of the numerator as a function of the loop momentum, while the others return the leading terms of a parametric Laurent expansion of the numerator. NINJA computes the parametric solutions of each multiple cut, performs the Laurent expansions via a simplified polynomial division between the (expansions of the) numerator and the denominators, and implements the subtractions at the coefficient level in order to get the unknown coefficients. These are then multiplied by the corresponding MI's. NINJA implements a wrapper of the ONELOOP library [30,31] which caches the values of computed integrals and allows for constant time lookups from their arguments. The library can also be used for the reduction of higher-rank integrands where the rank of a numerator can exceed the number of denominators by one. The simplified fit of the coefficients and the subtractions at coefficient level make the algorithm implemented in NINJA significantly lighter, faster and more stable than the original.

The first new phenomenological application of NINJA has been the computation of NLO QCD corrections to Higgs boson production in association with a top quark pair and a jet [14]. The possibility of exploiting the improved stability of the new algorithm has been especially important for the computation of the corresponding six-point virtual amplitude, given the presence of two mass scales as well as massive loop propagators which make traditional integrand reduction algorithms 


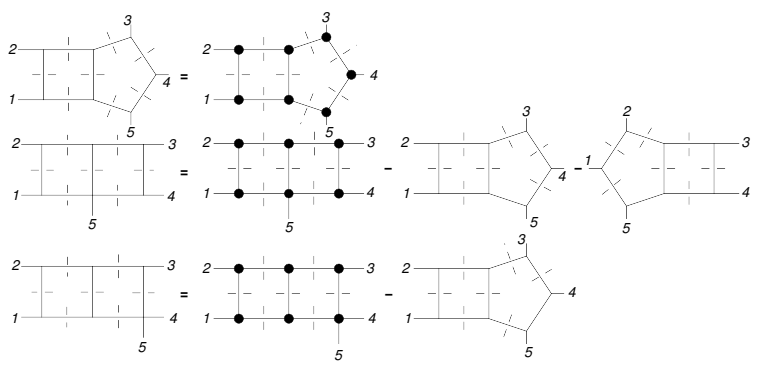

Figure 1: The eight- and seven-pole unitarity cuts of the pentabox graph, which are used to determine the corresponding residues.

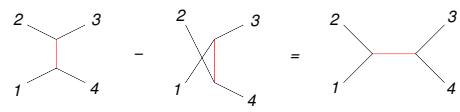

Figure 2: Graph representation of the Jacobi Identity at four points

numerically unstable. Indeed, for the highly non-trivial process under consideration, only a number of phase-space points of the order of one per mill were detected as unstable. All these points have been recovered using the tensorial reduction provided by GOLEM95 $[32,33]$.

\section{Application to Two-Loop Scattering Amplitudes in $\mathscr{N}=4 \mathrm{SYM}$}

The integrand reduction within the fit-on-the-cut approach has been combined with the colorkinematic duality [34] in order to construct the two-loop five-point amplitude for $\mathscr{N}=4$ super YangMills (sYM) [35].

The inputs for the integrand decomposition are the products of the trees to be sewn along the multiple cuts and the parametric forms of the residues. The former can be computed by adopting the super-amplitude formalism [36], while the latter were classified in [16].

The reduction of this amplitude begins from the eight-pole cuts, which are maximum cuts [11], and terminates after determining the residues at the seven-pole cuts. The absence of lower cuts is compatible with the property that $\mathscr{N}=4 \mathrm{sYM}$ integrands are linear in the loop-momenta. Representative steps of the reduction are given in Fig. 1.

Once the reduction is completed, one can equivalently construct a numerator function for the parent eight-denominator topologies, which captures the whole structure of the scattering amplitude. These numerators can be rearranged in a color-kinematic dual form by imposing additional constraints, referred to as BCJ equations, derived from the kinematic equivalent of the Jacobi Identity portrayed in Fig. 2. BCJ identities, beyond one-loop, imply a relation between the integrands of planar and non-planar topologies. The color-kinematic dual numerator for the eight-denominator planar diagram is represented in the first line of Fig.4. The key equations for the determination of the new numerators of the planar diagrams are depicted in Fig. 3, where one may notice the rising of a seven-denominator diagram, whose identification was not needed in the unitarity decomposition. In order to disentangle the contributions to the seven-pole cuts we use the BCJ equations which only involve the planar topologies displayed in the last two lines of Fig. 4. The obtained results are in agreement with [37]. 


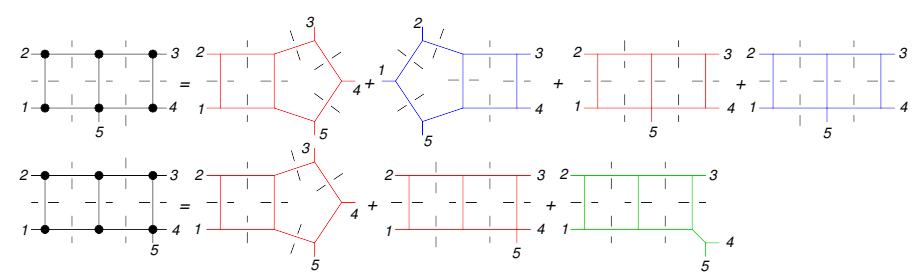

Figure 3: Each seven-pole unitarity cut is split into two contributions as indicated by the colors. The green diagram represents the new seven-denominator topology arising from the BCJ equations.

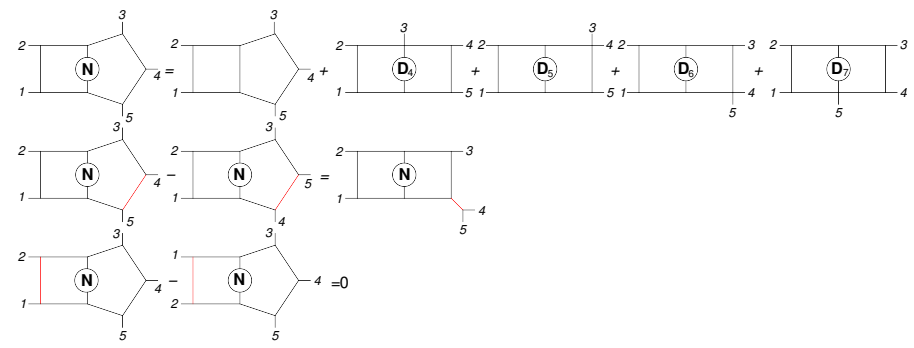

Figure 4: The first line shows the decomposition of the numerator $N$ of the planar topology, in terms of residues of eight-pole cuts and seven-pole cuts multiplied by the corresponding denominators $D_{i}$. The last two lines show the BCJ equations which are used to disentangle the seven-pole unitarity cuts.

\section{Divide-and-conquer approach}

The direct application of the integrand reduction formula of Eq. (2.4) on the numerator of an $l$ loop graph allows to perform the integrand decomposition algebraically by successive polynomial divisions, within what we call the divide-and-conquer approach [12]. At each step, the remainders of the divisions are identified with the residues of the corresponding set of denominators, while the quotients become the numerators of the lower-point integrands appearing on the r.h.s. of the formula, allowing thus to iterate the procedure. In this way, the decomposition of any integrand is obtained analytically, with a finite number of algebraic operations, without requiring the knowledge of the varieties of solutions of the multiple cuts, nor the one of the parametric form of the residues.

This algorithm has been implemented in a PYTHON package, which can perform the decomposition of any numerator using FORM [38] and MACAULAY2 [39] for the algebraic operations. It has been applied to the examples depicted in Fig. 5. Despite their simplicity, these show the broadness of applicability of the method which is not affected by the presence of massive propagators, non planar diagrams, higher powers of loop denominators or higher-rank contributions in the numerator.

\section{Conclusions}

We described a coherent framework for the decomposition of Feynman integrals, which can be applied at any loop order, regardless of the complexity of the integrand, the number of external legs or the presence of higher powers of loop denominators. This framework allows to easily derive well known results at one-loop order and extend them to higher loops. 


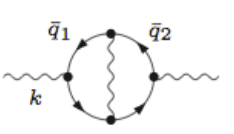

$(a)$

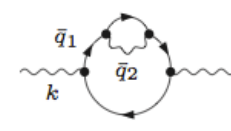

(b)

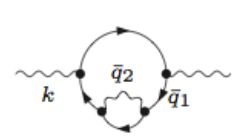

$(c)$

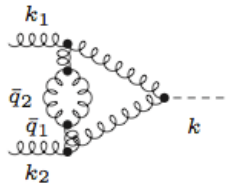

$(d)$

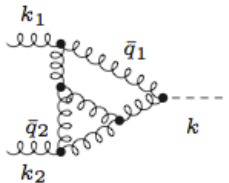

$(e)$

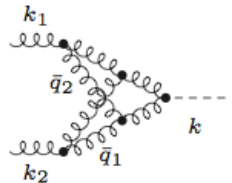

$(f)$

Figure 5: Examples of diagrams reduced using the divide-and-conquer approach.

In the one-loop case, we showed how the knowledge of the analytic structure of the integrands on the multiple cuts, and in particular their asymptotic behavior on the on-shell solutions, can be used to improve the analytic and semi-analytic reduction with the Laurent expansion method. Its implementation in the $\mathrm{C}++$ library NINJA provided a considerable gain in the speed and in the stability of the reduction.

At higher loops we presented the application of the fit-on-the-cut approach to 5-point amplitudes in $\mathscr{N}=4$ super Yang-Mills (sYM), with a unitarity-based construction of the integrands on the multiple cuts. We also described the application of the divide-and-conquer approach, which allows to perform the full decomposition with purely algebraic operations, without requiring the knowledge of the algebraic variety defined by the on-shell solutions. We applied it to simple examples, some of which cannot be addressed with other unitarity-based and integrand-reduction methods, due to the presence of higher powers of loop denominators in the integrands. Since it is based on the same principles used to constructively prove the existence of the integrand decomposition at all loops, the divide-and-conquer approach doesn't have the limitations of other methods and can be considered a more general integrand reduction algorithm.

\section{Acknowledgments}

The work of H.v.D., G.L., P.M., T.P. and U.S. was supported by the Alexander von Humboldt Foundation, in the framework of the Sofja Kovalevskaja Award Project "Advanced Mathematical Methods for Particle Physics", endowed by the German Federal Ministry of Education and Research. G.O. was supported in part by the National Science Foundation under Grant PHY-1068550.

\section{References}

[1] F. Cachazo, P. Svrcek and E. Witten, JHEP 0409 (2004) 006 [hep-th/0403047].

[2] R. Britto, F. Cachazo and B. Feng, Nucl. Phys. B 715 (2005) 499 [hep-th/0412308].

[3] R. Britto, F. Cachazo, B. Feng and E. Witten, Phys. Rev. Lett. 94 (2005) 181602 [hep-th/0501052].

[4] Z. Bern, L. J. Dixon, D. C. Dunbar and D. A. Kosower, Nucl. Phys. B 425 (1994) 217 [hep-ph/9403226].

[5] R. Britto, F. Cachazo and B. Feng, Nucl. Phys. B 725 (2005) 275 [hep-th/0412103].

[6] G. Ossola, C. G. Papadopoulos and R. Pittau, Nucl. Phys. B 763 (2007) 147.

[7] R. K. Ellis, W. T. Giele and Z. Kunszt, JHEP 0803 (2008) 003. 
[8] P. Mastrolia and G. Ossola, JHEP 1111 (2011) 014.

[9] S. Badger, H. Frellesvig and Y. Zhang, JHEP 1204 (2012) 055.

[10] Y. Zhang, JHEP 1209 (2012) 042.

[11] P. Mastrolia, E. Mirabella, G. Ossola and T. Peraro, Phys. Lett. B 718 (2012) 173.

[12] P. Mastrolia, E. Mirabella, G. Ossola and T. Peraro, arXiv:1307.5832 [hep-ph].

[13] P. Mastrolia, E. Mirabella and T. Peraro, JHEP 1206 (2012) 095 [Erratum-ibid. 1211A (2012) 128].

[14] H. van Deurzen, G. Luisoni, P. Mastrolia, E. Mirabella, G. Ossola and T. Peraro, Phys. Rev. Lett. 111 (2013) 171801.

[15] S. Badger, H. Frellesvig and Y. Zhang, arXiv:1310.1051 [hep-ph].

[16] P. Mastrolia, E. Mirabella, G. Ossola and T. Peraro, Phys. Rev. D 87 (2013) 085026.

[17] S. Badger, H. Frellesvig and Y. Zhang, JHEP 1208 (2012) 065.

[18] P. Mastrolia, G. Ossola, T. Reiter and F. Tramontano, JHEP 1008 (2010) 080.

[19] H. van Deurzen, N. Greiner, G. Luisoni, P. Mastrolia, E. Mirabella, G. Ossola, T. Peraro and J. F. von Soden-Fraunhofen et al., Phys. Lett. B 721 (2013) 74.

[20] G. Cullen, H. van Deurzen, N. Greiner, G. Luisoni, P. Mastrolia, E. Mirabella, G. Ossola and T. Peraro et al., Phys. Rev. Lett. 111 (2013) 131801.

[21] D. Forde, Phys. Rev. D 75 (2007) 125019.

[22] S. D. Badger, JHEP 0901 (2009) 049.

[23] G. Ossola, C. G. Papadopoulos and R. Pittau, JHEP 0803 (2008) 042.

[24] P. Mastrolia, G. Ossola, C. G. Papadopoulos and R. Pittau, JHEP 0806 (2008) 030.

[25] G. Cullen, N. Greiner, G. Heinrich, G. Luisoni, P. Mastrolia, G. Ossola, T. Reiter and F. Tramontano, Eur. Phys. J. C 72 (2012) 1889.

[26] P. Mastrolia, in these proceedings.

[27] T. Gehrmann, N. Greiner and G. Heinrich, in these proceedings, arXiv:1311.4754 [hep-ph].

[28] G. Luisoni, in these proceedings.

[29] J. Schlenk, G. Heinrich, J. Winter, in these proceedings.

[30] A. van Hameren, Comput. Phys. Commun. 182 (2011) 2427.

[31] A. van Hameren, C. G. Papadopoulos and R. Pittau, JHEP 0909 (2009) 106.

[32] T. Binoth, J. -P. .Guillet, G. Heinrich, E. Pilon and T. Reiter, Comput. Phys. Commun. 180 (2009) 2317.

[33] G. Cullen, J. P. .Guillet, G. Heinrich, T. Kleinschmidt, E. Pilon, T. Reiter and M. Rodgers, Comput. Phys. Commun. 182 (2011) 2276.

[34] Z. Bern, J. J. M. Carrasco and H. Johansson, Phys. Rev. D 78 (2008) 085011.

[35] U. Schubert, Diplomarbeit (2013) .

[36] J. M. Drummond, J. Henn, G. P. Korchemsky and E. Sokatchev, Nucl. Phys. B 869 (2013) 452.

[37] J. J. .Carrasco and H. Johansson, Phys. Rev. D 85 (2012) 025006.

[38] J. A. M. Vermaseren, math-ph/0010025.

[39] D. R. Grayson and M. E. Stillman, Available at http://www.math.uiuc.edu/Macaulay2. 